\title{
Reliable Solution of a Unilateral Frictionless Contact Problem in Quasi-Coupled Thermo-Elasticity with Uncertain Input Data
}

\author{
Ivan Hlaváček ${ }^{1}$ and Jiří Nedoma ${ }^{1}$ \\ Institute of Computer Science, Academy of Sciences of the Czech Republic \\ Pod Vodárenskou věží 2, 18207 Prague 8, Czech Republic
}

\begin{abstract}
A unilateral contact problem without friction in quasi-coupled thermo-elasticity and with uncertain input data is analysed. The worst scenario method is used to find the most "dangerous" admissible input data.
\end{abstract}

\section{Introduction}

In this contribution we deal with contact problems without friction (see [4], [5], [6]) in quasi-coupled thermo-elasticity considering uncertain input data representing extension of problems solved in [5] and [6]. By uncertain input data we mean physical coefficients, right-hand sides, etc., which cannot be determined uniquely but only in some intervals determined by the measurements. The reliable solution is defined as the worst among a set of possible solutions, and the degree of badness is measured by a criterion-functional (see [1]). The main aim of our contribution will be to find maximal values of this functional. We prove the solvability of the corresponding maximization (worst scenario) problems.

\section{Formulation of the Problem}

Let us assume a union $\Omega$ of bounded domains $\Omega^{\iota}, \iota=1, \ldots, s$, with Lipschitz boundaries $\partial \Omega^{\iota}$, occupied by elastic bodies such that $\Omega=\bigcup_{\iota=1}^{s} \Omega^{\iota} \subset R^{2}$. Let the boundary $\partial \Omega=\cup_{\iota=1}^{s} \partial \Omega^{\iota}$ consist of three disjoint parts $\Gamma_{\tau}, \Gamma_{u}$ and $\Gamma_{c}$, such that $\partial \Omega=\bar{\Gamma}_{\tau} \cup \bar{\Gamma}_{u} \cup \bar{\Gamma}_{c}, \Gamma_{c}=\bigcup_{k, l} \Gamma^{k l}, \Gamma^{k l}=\partial \Omega^{k} \cap \partial \Omega^{l}, 1 \leq k, l \leq s$, for $k \neq l$, and $\bar{\Gamma}_{\tau}, \bar{\Gamma}_{u}, \bar{\Gamma}_{c}$ denotes the closures in $\partial \Omega$.

Let the heat sources $W^{\iota}$, the prescribed temperature $T_{1}$, the body forces $\mathbf{F}$, the surface forces $\mathbf{P}$, displacements $\mathbf{u}_{0}$, elastic coefficients $c_{i j k l}$, coefficients of thermal expansion $\beta_{i j}$ and the reference temperature $T_{0}$ be given. Throughout the paper we use the summation convention, i.e. a repeated index implies summation from 1 to 2 . Furthermore, $\mathbf{n}^{k}=\left(n_{i}^{k}\right), i=1,2,1 \leq k \leq s$, denotes the unit normal with respect to $\partial \Omega^{k}, \mathbf{n}^{k}=-\mathbf{n}^{l}$ on $\Gamma^{k l}$. Assume that $\kappa^{\iota}$ and $C^{\iota}$ are positive definite symmetric matrix functions, 


$$
\begin{gathered}
0<\kappa_{0}^{\iota} \leq \kappa_{i j}^{\iota} \zeta_{i} \zeta_{j}|\zeta|^{-2} \leq \kappa_{1}^{\iota}<+\infty \text { for a.a. } \mathbf{x} \in \Omega^{\iota}, \zeta \in R^{2}, \\
0<c_{0}^{\iota} \leq c_{i j k l}^{\iota} \xi_{i j} \xi_{k l}|\boldsymbol{\xi}|^{-2} \leq c_{1}^{\iota}<+\infty \text { for a.a. } \mathbf{x} \in \Omega^{\iota}, \boldsymbol{\xi} \in R^{4}, \xi_{i j}=\xi_{i j},
\end{gathered}
$$

where $\kappa_{0}^{\iota}, \kappa_{1}^{\iota}, c_{0}^{\iota}, c_{1}^{\iota}$ are constants independent of $\mathbf{x} \in \Omega^{\iota}$. Let $\kappa_{i j}^{\iota} \in L^{\infty}\left(\Omega^{\iota}\right)$, $W^{\iota} \in L^{2}\left(\Omega^{\iota}\right), T_{1}^{\iota} \in H^{1}\left(\Omega^{\iota}\right), T_{1}^{k}=T_{1}^{l}$ on $\bigcup_{k, l} \Gamma^{k l}, c_{i j k l}^{\iota} \in L^{\infty}\left(\Omega^{\iota}\right), F_{i}^{\iota} \in L^{2}\left(\Omega^{\iota}\right)$, $P_{i} \in L^{2}\left(\Gamma_{\tau}\right), \beta_{i j}^{\iota} \in L^{\infty}\left(\Omega^{\iota}\right), \mathbf{u}_{0}^{\iota} \in\left[H^{1}\left(\Omega^{\iota}\right)\right]^{2}$.

We will deal with the following problem:

Problem $(\mathcal{P})$ : Find a pair of functions $(T, \mathbf{u})$ satisfying

$$
\begin{aligned}
& \frac{\partial}{\partial x_{i}}\left(\kappa_{i j}^{\iota} \frac{\partial T^{\iota}}{\partial x_{j}}\right)+W^{\iota}=0, \frac{\partial}{\partial x_{j}} \tau_{i j}\left(\mathbf{u}^{\iota}, T^{\iota}\right)+F_{i}^{\iota}=0 \text { in } \Omega^{\iota}, 1 \leq \iota \leq s, i=1,2 \\
& \tau_{i j}\left(\mathbf{u}^{\iota}, T^{\iota}\right)=c_{i j k l}^{\iota} e_{k l}\left(\mathbf{u}^{\iota}\right)-\beta_{i j}^{\iota}\left(T^{\iota}-T_{0}^{\iota}\right) \text { in } \Omega^{\iota}, 1 \leq \iota \leq s, i=1,2 \\
& \kappa_{i j} \frac{\partial T}{\partial x_{j}} n_{i}=0, \quad \mathbf{u}=\mathbf{u}_{0} \text { on } \Gamma_{u}, \\
& T=T_{1}, \quad \tau_{i j}(\mathbf{u}, T) n_{j}=P_{i} \text { on } \Gamma_{\tau}, \\
& T^{k}=T^{l}, \quad\left(\kappa_{i j} \frac{\partial T}{\partial x_{j}} n_{i}\right)^{k}-\left(\kappa_{i j} \frac{\partial T}{\partial x_{j}} n_{i}\right)^{l}=0 \text { on } \bigcup_{k, l} \Gamma^{k l}, 1 \leq k, l \leq s, \\
& u_{n}^{k}-u_{n}^{l} \leq 0, \quad \tau_{n}^{k} \leq 0, \quad\left(u_{n}^{k}-u_{n}^{l}\right) \tau_{n}^{k}=0 \text { on } \bigcup_{k, l} \Gamma^{k l}, 1 \leq k, l \leq s, \\
& \tau_{t}^{k}=-\tau_{t}^{l}=0 \text { on } \bigcup_{k, l} \Gamma^{k l}, 1 \leq k, l \leq s,
\end{aligned}
$$

where $e_{i j}(\mathbf{u})=\frac{1}{2}\left(\frac{\partial u_{i}}{\partial x_{j}}+\frac{\partial u_{j}}{\partial x_{i}}\right), u_{n}^{k}=u_{i}^{k} n_{i}^{k}, u_{n}^{l}=u_{i}^{l} n_{i}^{l}=-u_{i}^{k} n_{i}^{k}$ (no sum over $k$ or $l), u_{t}^{k}=\left(u_{t i}^{k}\right), u_{t i}^{k}=u_{i}^{k}-u_{n}^{k} n_{i}^{k}, u_{t}^{l}=\left(u_{t i}^{l}\right), u_{t i}^{l}=u_{i}^{l}-u_{n}^{l} n_{i}^{l}, i=1,2, \tau_{n}^{k}=$ $\tau_{i j}^{k} n_{i}^{k} n_{j}^{k}, \tau_{t}^{k}=\left(\tau_{t i}^{k}\right), \tau_{t i}^{k}=\tau_{i j}^{k} n_{j}^{k}-\tau_{n}^{k} n_{i}^{k}, \tau_{n}^{l}=\tau_{i j}^{l} n_{i}^{l} n_{j}^{l}, \tau_{t}^{l}=\left(\tau_{t i}^{l}\right), \tau_{t i}^{l}=\tau_{i j}^{l} n_{j}^{l}-\tau_{n}^{l} n_{i}^{l}$.

Since the stress and strain tensors and coefficient of thermal expansion are symmetric then the entries of any symmetric $3 \times 3$ matrices $\left\{\tau_{i j}\right\}$ can be rewritten in the vector notation $\left\{\tau_{j}\right\}, j=1,2,3$ and similarly the symmetric matrices $\left\{e_{i j}\right\},\left\{\beta_{i j}\right\}$ by vectors $\left\{e_{j}\right\},\left\{\beta_{j}\right\}$. Then (2) can be rewritten as

$$
\tau_{i}\left(\mathbf{u}^{\iota}, T^{\iota}\right)=\sum_{j=1}^{3} A_{i j}^{\iota} e_{j}\left(\mathbf{u}^{\iota}\right)-\beta_{i}^{\iota}\left(T^{\iota}-T_{0}^{\iota}\right) \text { in } \Omega^{\iota}, 1 \leq \iota \leq s, 1 \leq i, j \leq 3,
$$

where $A^{\iota}$ is a symmetric $3 \times 3$ matrix, $A_{i k}^{\iota} \in L^{\infty}\left(\Omega^{\iota}\right), \iota=1, \ldots, s$. Since $\tau_{i j} e_{i j}=$ $\sum_{i=1}^{2} \tau_{i} e_{i}+2 \tau_{3} e_{3}$, we can write 


$$
c_{i j k l}^{\iota} e_{i j} e_{k l}=\sum_{i, j=1}^{3} B_{i j}^{\iota} e_{i} e_{j},
$$

where $B^{\iota}$ is a symmetric $3 \times 3$ matrix such that $B_{i j}^{\iota}=A_{i j}^{\iota}$ for $i, j=1,2$, $B_{i j}^{\iota}=\frac{3}{2} A_{i j}^{\iota}$ for $i=1,2, j=3$ and $B_{i j}^{\iota}=2 A_{i j}^{\iota}$ for $i, j=3$.

In what follows, we denote

$$
\begin{aligned}
& W_{1}=\Pi_{\iota=1}^{s} H^{1}\left(\Omega^{\iota}\right), \quad\|w\|_{W_{1}}=\left(\sum_{\iota \leq s}\left\|w^{\iota}\right\|_{1, \Omega^{\iota}}^{2}\right)^{\frac{1}{2}}, \\
& W=\Pi_{\iota=1}^{s}\left[H^{1}\left(\Omega^{\iota}\right)\right]^{2},\|v\|_{W}=\left(\sum_{\iota \leq s} \sum_{i \leq 2}\left\|v_{i}^{\iota}\right\|_{1, \Omega^{\iota}}^{2}\right)^{\frac{1}{2}}, \\
& V_{1}=\left\{z \mid z \in W_{1}, z=0 \text { on } \Gamma_{\tau}, z^{k}=z^{l} \text { on } \bigcup_{k, l} \Gamma^{k l}\right\}, \\
& V=\left\{\mathbf{v} \mid \mathbf{v} \in W, \mathbf{v}=0 \text { on } \Gamma_{u}\right\}, \quad K=\left\{\mathbf{v} \mid \mathbf{v} \in V, v_{n}^{k}-v_{n}^{l} \leq 0 \text { on } \bigcup_{k, l} \Gamma^{k l}\right\} .
\end{aligned}
$$

Definition 1. We say that the pair of functions $T$ and $\mathbf{u}$ is a weak solution of problem $(\mathcal{P})$, if $T-T_{1} \in V_{1}$,

$$
\begin{aligned}
& b(T, z)=s(z) \quad \forall z \in V_{1} \\
& \mathbf{u}-\mathbf{u}_{0} \in K \\
& a(\mathbf{u}, \mathbf{v}-\mathbf{u}) \geq S(\mathbf{v}-\mathbf{u}, T) \quad \forall \mathbf{v} \in \mathbf{u}_{0}+K
\end{aligned}
$$

where

$$
\begin{aligned}
b(T, z) & =\sum_{\iota=1}^{s} \int_{\Omega^{\iota}} \kappa_{i j}^{\iota} \frac{\partial T^{\iota}}{\partial x_{i}} \frac{\partial z^{\iota}}{\partial x_{j}} d \mathbf{x}, \quad s(z)=\sum_{\iota=1}^{s} \int_{\Omega^{\iota}} W^{\iota} z^{\iota} d \mathbf{x}, \\
a(\mathbf{u}, \mathbf{v}) & =\sum_{\iota=1}^{s} \int_{\Omega^{\iota}} \sum_{i, j=1}^{3} B_{i j}^{\iota} e_{i}\left(\mathbf{u}^{\iota}\right) e_{j}\left(\mathbf{v}^{\iota}\right) d \mathbf{x}, \\
S(\mathbf{v}, T) & =\sum_{\iota=1}^{s} \int_{\Omega^{\iota}} F_{i}^{\iota} v_{i}^{\iota} d \mathbf{x}+\int_{\Gamma_{\tau}} P_{i} v_{i} d s-\sum_{\iota=1}^{s} \int_{\Omega^{\iota}} \beta_{i}^{\iota}\left(T^{\iota}-T_{0}^{\iota}\right) v_{i}^{\iota} d \mathbf{x} .
\end{aligned}
$$

Remark 1. In $S(\mathbf{v}, T)$ we insert the weak solution $T$ of (9). Moreover, we assume that $\mathbf{u}_{0}$ satisfies

$$
u_{0 n}^{k}-u_{0 n}^{l}=0 \quad \text { on } \bigcup_{k, l} \Gamma^{k l}
$$




\section{Worst Scenario Method for Uncertain Input Data}

Let us assume that the input data

$$
A=\left\{B^{\iota}, \kappa^{\iota}, F_{i}^{\iota}, W^{\iota}, \beta_{i}^{\iota}, P_{i}, u_{0 i}, T_{1}, \iota=1, \ldots, s, i=1,2\right\}
$$

are uncertain, and belong to some sets of admissible data, i.e.

$$
\begin{aligned}
& A \in U_{a d} \Leftrightarrow B^{\iota} \in U_{a d}^{B^{\iota}}, \kappa^{\iota} \in U_{a d}^{\kappa^{\iota}}, F_{i}^{\iota} \in U_{a d}^{F_{i}^{\iota}}, W^{\iota} \in U_{a d}^{W^{\iota}}, \beta_{i}^{\iota} \in U_{a d}^{\beta_{i}^{\iota}}, \\
& P \in U_{a d}^{P_{i}}, u_{0 i} \in U_{a d}^{u_{0 i}}, T_{1} \in U_{a d}^{T_{1}} .
\end{aligned}
$$

We will assume that all the bodies $\Omega^{\iota}$ are piecewise homogeneous, so that partitions of $\bar{\Omega}^{\iota}$ exist such that

$$
\begin{aligned}
& \bar{\Omega}^{\iota}=\bigcup_{j=1}^{r^{\iota}} \bar{\Omega}_{j}^{\iota}, \Omega_{j}^{\iota} \cap \Omega_{k}^{\iota}=\oslash \text { for } j \neq k, 1 \leq \iota \leq s, \\
& \Gamma^{k l}=\bigcup_{q=1}^{Q_{k l}} \bar{\Gamma}_{q}^{k l}, \Gamma_{q}^{k l} \cap \Gamma_{p}^{k l}=\oslash \text { for } q \neq p, \forall k, l .
\end{aligned}
$$

Let the data $B^{\iota}, \kappa^{\iota}, \mathbf{F}^{\iota}, W^{\iota}, \beta^{\iota}$ be piecewise constant with respect to the corresponding partitioning (15) and let us denote

$$
\Gamma_{u}^{\iota}=\Gamma_{u} \cap \partial \Omega^{\iota}, \quad \iota=1, \ldots, s \text { and } \Gamma_{\tau}^{\iota}=\Gamma_{\tau} \cap \partial \Omega^{\iota}, \iota \leq s .
$$

Further, we define the sets of admissible matrices:

$$
\begin{aligned}
& U_{a d}^{B^{\iota}}=\left\{3 \times 3 \text { symmetric matrices } B^{\iota}: \underline{B}_{i k}^{\iota}(j) \leq B_{\left.i k\right|_{\Omega_{j}^{\iota}}}=\text { const. } \leq \bar{B}_{i k}^{\iota}(j),\right. \\
& \left.j \leq r^{\iota}, i, k=1, \ldots, 3\right\}
\end{aligned}
$$

where $\underline{B}^{\iota}(j)$ and $\bar{B}^{\iota}(j)$ are given $3 \times 3$ symmetric matrices, $\iota=1, \ldots, s$, and let there exist positive constants $c_{B}^{\iota}(j)$ such that

$$
\begin{aligned}
& \lambda_{\min }\left(\frac{1}{2}\left(\underline{B}^{\iota}(j)+\bar{B}^{\iota}(j)\right)\right)-\rho\left(\frac{1}{2}\left(\bar{B}^{\iota}(j)-\underline{B}^{\iota}(j)\right)\right) \equiv c_{B}^{\iota}(j) \\
& \text { for } j=1, \ldots, r^{\iota}, \iota=1, \ldots, s,
\end{aligned}
$$

where $\lambda_{\min }$ and $\rho$ denotes the minimal eigenvalue and the spectral radius, respectively,

$$
\begin{aligned}
& U_{a d}^{\kappa^{\iota}}=\left\{2 \times 2 \text { symmetric matrices } \kappa^{\iota}: \underline{\kappa}_{i k}^{\iota}(j) \leq \kappa_{\left.i k\right|_{\Omega_{j}^{\iota}} ^{\iota}}=\text { const. } \leq \bar{\kappa}_{i k}^{\iota}(j),\right. \\
& \left.j \leq r^{\iota}, i, k \leq 2\right\}
\end{aligned}
$$


where $\underline{\kappa}^{\iota}(j)$ and $\bar{\kappa}^{\iota}(j)$ are given $2 \times 2$ symmetric matrices, $j=1, \ldots, r^{\iota}, \iota=$ $1, \ldots, s$, and let there exist positive constants $c_{B}^{\iota}(j)$ such that

$$
\lambda_{\min }\left(\frac{1}{2}\left(\underline{\kappa}^{\iota}(j)+\bar{\kappa}^{\iota}(j)\right)\right)-\rho\left(\frac{1}{2}\left(\bar{\kappa}^{\iota}(j)-\underline{\kappa}^{\iota}(j)\right)\right) \equiv c_{\kappa}^{\iota}(j) \text { for } j \leq r^{\iota}, \iota \leq s,
$$

where $\lambda_{\min }$ and $\rho$ denotes the minimal eigenvalue and the spectral radius, respectively. If (18) and (19) are satisfied, then the matrices $B^{\iota}(j) \equiv B_{\mid \Omega_{j}^{\iota}}$ are positive definite for any $B^{\iota} \in U_{a d}^{B^{\iota}}, \iota=1, \ldots, s$ and any $j \leq r^{\iota}$ (see [8]) and the matrices $\kappa^{\iota}(j)=\kappa_{\mid \Omega_{j}^{\iota}}$ are positive definite for any $\kappa^{\iota} \in U_{a d}^{\kappa^{\iota}}, \iota \leq s, j \leq r^{\iota}$.

Furthermore, we define

$$
U_{a d}^{F_{i}^{\iota}}=\left\{f \in L^{\infty}(\Omega): \underline{F}_{i}^{\iota}(j) \leq f_{\mid \Omega_{j}^{\iota}}=\text { const. } \leq \bar{F}_{i}^{\iota}(j), j \leq r^{\iota}\right\},
$$

for $i \leq 2, \iota \leq s$, where $\underline{F}_{i}^{\iota}(j)$ and $\bar{F}_{i}^{\iota}(j)$ are given constants;

$$
U_{a d}^{W^{\iota}}=\left\{w \in L^{\infty}(\Omega): \underline{W}^{\iota}(j) \leq w_{\mid \Omega_{j}^{\iota}}=\text { const. } \leq \bar{W}^{\iota}(j), j \leq r^{\iota}\right\}
$$

for $\iota \leq s$, where $\underline{W^{\iota}}(j)$ and $\bar{W}^{\iota}(j)$ are given constants;

$$
U_{a d}^{T_{1}}=\left\{\mathcal{T} \in L^{\infty}\left(\Gamma_{\tau}\right): \underline{T}_{1}(\iota) \leq \mathcal{T}_{\mid \Gamma_{\tau}^{\iota}}=\text { const. } \leq \bar{T}_{1}(\iota), \iota \leq s\right\},
$$

where $\underline{T}_{1}(\iota)$ and $\bar{T}_{1}(\iota)$ are given constants;

$$
U_{a d}^{u_{0 i}}=\left\{u \in L^{\infty}\left(\Gamma_{u}\right): \underline{u}_{0 i}(\iota) \leq u_{\mid \Gamma_{u}^{\iota}}=\text { const. } \leq \bar{u}_{0 i}(\iota), \iota \leq s\right\},
$$

where $\underline{u}_{0 i}(\iota)$ and $\bar{u}_{0 i}(\iota), i=1,2$, are given constants;

$$
U_{a d}^{P_{i}}=\left\{p \in L^{\infty}\left(\Gamma_{\tau}\right): \underline{P}_{i}(\iota) \leq p_{\mid \Gamma_{\tau}^{\iota}}=\text { const. } \leq \bar{P}_{i}(\iota), \iota \leq s\right\},
$$

where $\underline{P}_{i}(\iota)$ and $\bar{P}_{i}(\iota), i=1,2$, are given constants;

$$
U_{a d}^{\beta_{i}^{\iota}}=\left\{b \in L^{\infty}(\Omega): \underline{\beta}_{i}^{\iota}(j) \leq b_{\mid \Omega_{j}^{\iota}}=\text { const. } \leq \bar{\beta}_{i}^{\iota}(j), j \leq r^{\iota}\right\},
$$

for $i \leq 3, \iota \leq s$, where $\underline{\beta}_{i}^{\iota}(j)$ and $\bar{\beta}_{i}^{\iota}(j)$ are given constants.

Finally, we define the set of admissible data by

$$
\begin{aligned}
U_{a d}= & \sqcap_{\iota \leq s} U_{a d}^{B^{\iota}} \times \Pi_{\iota \leq s} U_{a d}^{\kappa^{\iota}} \times \Pi_{\iota \leq s, i \leq 2} U_{a d}^{F_{i}^{\iota}} \times \Pi_{\iota \leq s} U_{a d}^{W^{\iota}} \times \\
& \times \Pi_{\iota \leq s, i \leq 2} U_{a d}^{\beta_{i}^{\iota}} \times \Pi_{i \leq 2} U_{a d}^{P_{i}} \times \Pi_{i \leq 2} U_{a d}^{u_{0 i}} \times \Pi_{\iota \leq s} U_{a d}^{T_{1}} .
\end{aligned}
$$

Further, instead of $b(T, z), a(\mathbf{u}, \mathbf{v}), s(z), S(\mathbf{v}, T)$ we will write $b(A ; T, z), a(A ; \mathbf{u}, \mathbf{v})$, $s(A ; z), S(A ; \mathbf{v}, T)$ for any $A \in U_{a d}$.

The next results are parallel to those of [3] for the general case with friction. 
Lemma 1. There exist positive constants $c_{i}, i=0,1, \ldots, 5$ independent of $A \in$ $U_{a d}$, such that

$$
\begin{aligned}
b(A ; z, z) & \geq c_{0}\|z\|_{W^{1}}^{2} \quad \forall z \in V_{1} \\
|b(A ; z, y)| & \leq c_{1}\|z\|_{W^{1}}\|y\|_{W^{1}} \quad \forall z, y \in W_{1}, \\
a(A ; \mathbf{v}, \mathbf{v}) & \geq c_{2}\|\mathbf{v}\|_{W}^{2} \quad \forall \mathbf{v} \in V \\
|a(A ; \mathbf{v}, \mathbf{w})| & \leq c_{3}\|\mathbf{v}\|_{W}\|\mathbf{w}\|_{W} \quad \forall \mathbf{v}, \mathbf{w} \in W \\
|s(A ; z)| & \leq c_{4}\|z\|_{0, \Omega} \quad \forall z \in V_{1} \\
|S(A ; \mathbf{v}, T)| & \leq c_{5}\left(\|\mathbf{v}\|_{0, \Omega}+\|\mathbf{v}\|_{0, \Gamma_{\tau}}+\left\|T-T_{0}\right\|_{0, \Omega}\|\mathbf{v}\|_{W}\right) \quad \forall \mathbf{v}, \mathbf{w} \in W .
\end{aligned}
$$

Proposition 1. There exists a unique weak solution $(T(A), \mathbf{u}(A))$ of the problem $(\mathcal{P})$ for any $A \in U_{a d}$. Moreover, $\|T(A)\|_{W_{1}} \leq c$, where $c$ is independent of $A$.

To find the most "dangerous" input data $A$ in the set $U_{a d}$, we will introduce a criterion, i.e. defined a functional, which depends on the solution $(T(A), \mathbf{u}(A))$ of the problem $(\mathcal{P})$. Such criteria can be as follows:

Let $G_{r} \subset \bigcup_{\iota \leq s} \Omega^{\iota}, r=1, \ldots, \bar{r}$, be subdomains adjacent to the boundaries $\partial \Omega^{\iota}$. Then we define

$$
\Phi_{1}(T)=\max _{r \leq \bar{r}} \varphi_{r}(T)=\max _{r \leq \bar{r}}\left[\left(\operatorname{meas}_{2} G_{r}\right)^{-1} \int_{G_{r}} T d \mathbf{x}\right] ;
$$

let $G_{r}^{\prime} \subset \Gamma_{u}, r \leq \bar{r}$ and

$$
\Phi_{2}(T)=\max _{r \leq \bar{r}} \psi_{r}(T)=\max _{r \leq \bar{r}}\left[\left(\operatorname{meas}_{1} G_{r}^{\prime}\right)^{-1} \int_{G_{r}^{\prime}} T d s\right] ;
$$

and

$$
\Phi_{3}(\mathbf{u})=\max _{r \leq \bar{r}} \chi_{r}(\mathbf{u})=\max _{r \leq \bar{r}}\left[\left(\operatorname{meas}_{2} G_{r}\right)^{-1} \int_{G_{r}} u_{i} n_{i}\left(X_{r}\right) d \mathbf{x}\right] ;
$$

where $\mathbf{n}\left(X_{r}\right)$ is the unit outward normal at a fixed point $X_{r} \in \partial \Omega^{\iota} \cap \partial G_{r}$ (if $\left.G_{r} \subset \Omega^{\iota}\right)$ to the boundary $\partial \Omega^{\iota}$;

$$
\Phi_{4}(\mathbf{u})=\max _{r \leq \bar{r}} \chi_{r}^{\prime}(\mathbf{u})=\max _{r \leq \bar{r}}\left[\left(\operatorname{meas}_{1} G_{r}^{\prime}\right)^{-1} \int_{G_{r}^{\prime}} u_{i} n_{i}\left(X_{r}\right) d s\right] ;
$$

where $G_{r}^{\prime}=\bigcup_{\iota \leq s} \partial \Omega^{\iota} \backslash \Gamma_{u}$. Since the weak solution $\mathbf{u}(A)$ of our problem $(10)$ depends on $T(A)$, then $\mathbf{u}(A)=\mathbf{u}(A ; T(A))$ and instead of $\Phi_{i}(\mathbf{u})$ we write $\Phi_{i}(A ; \mathbf{u}, T)$. Thus we may define

$$
\Phi_{5}(A ; u, T)=\max _{r \leq \bar{r}} \omega_{r}(A ; u, T)=\max _{r \leq \bar{r}}\left[\left(\operatorname{meas}_{2} G_{r}\right)^{-1} \int_{G_{r}} I_{2}^{2}(\tau(A ; \mathbf{u}, T)) d \mathbf{x}\right]
$$


here $I_{2}(\tau)=\left(\sum_{i, j=1}^{3} \tau_{i j}^{D} \tau_{i j}^{D}\right)^{\frac{1}{2}}$ is the intensity of shear stress, where $\tau_{i j}^{D}=\tau_{i j}$ $\frac{1}{3} \tau_{k k} \delta_{i j}$ and $\tau(A ; \mathbf{u}, T)$ is defined by (2). Finally, we may choose

$$
\Phi_{6}(A ; u, T)=\max _{r \leq \bar{r}} \mu_{r}(A ; u, T)=\max _{r \leq \bar{r}}\left[\left(\operatorname{meas}_{2} G_{r}\right)^{-1} \int_{G_{r}}\left(-\tau_{n}(A ; \mathbf{u}, T)\right) d \mathbf{x}\right]
$$

where $G_{r}$ is a small subdomain adjacent to $\Gamma_{c}$.

Now we formulate the worst scenario problems as follows: find

$$
A^{0 i}=\arg \max _{A \in U_{a d}} \Phi_{i}(T(A)), \quad i=1,2
$$

and

$$
A^{0 i}=\arg \max _{A \in U_{a d}} \Phi_{i}(\mathbf{u}(A), T(A)), \quad i=3,4,5,6
$$

where $(T(A), \mathbf{u}(A))$ is weak solution of the problem $(\mathcal{P})$.

\section{Stability of Weak Solutions}

To prove the solvability of worst scenario problems (41), (42), we have to study the mapping $A \mapsto T(A), A \mapsto \mathbf{u}(A, T(A))$. We introduce the decomposition of $A \in U_{a d}$ as $A=\left\{A^{\prime}, A^{\prime \prime}\right\}$, where

$A^{\prime}=\left\{\sqcap_{\iota \leq s} \Pi_{j \leq r^{\iota}} \kappa^{\iota}(j), \sqcap_{\iota \leq s} \sqcap_{j \leq r^{\iota}} W^{\iota}(j), \Pi_{\iota \leq s} T_{1}^{\iota}\right\}, A^{\prime} \in R^{p_{1}}, p_{1}=4 \sum_{\iota \leq s} r^{\iota}+s$,

and

$$
\begin{aligned}
& A^{\prime \prime}=\left\{\sqcap_{\iota \leq s} \sqcap_{j \leq r^{\iota}} B^{\iota}(j), \sqcap_{\iota \leq s} \sqcap_{j \leq r^{\iota}} \mathbf{F}^{\iota}(j), \Pi_{\iota \leq s} \mathbf{P}^{\iota}, \Pi_{\iota \leq s} \mathbf{u}_{0}^{\iota}, \Pi_{\iota \leq s} \Pi_{j \leq r^{\iota}} \beta^{\iota}(j)\right\}, \\
& A^{\prime \prime} \in R^{p_{2}}, \quad p_{2}=\left(\sum_{\iota \leq s} r^{\iota}\right)[9+2(1+2 s)] .
\end{aligned}
$$

We are going to show the continuity of the mappings $A^{\prime} \mapsto T\left(A^{\prime}\right), A \mapsto$ $\mathbf{u}\left(A, T\left(A^{\prime}\right)\right)$ for $A^{\prime} \in U_{a d}^{\prime}=\Pi_{\iota \leq s} U_{a d}^{\kappa^{\iota}} \times \Pi_{\iota \leq s} U_{a d}^{W^{\iota}} \times U_{a d}^{T_{1}^{\iota}}$ and $A^{\prime \prime} \in U_{a d}^{\prime \prime}=$ $\Pi_{\iota \leq s} U_{a d}^{B^{\iota}} \times \Pi_{\iota \leq s, i \leq 2} U_{a d}^{F_{i}^{\iota}} \times \Pi_{\iota \leq s, i \leq 2} U_{a d}^{\beta_{i}^{\iota}} \times \Pi_{i \leq 2} U_{a d}^{P_{i}} \times \Pi_{i \leq 2} U_{a d}^{u_{0 i}}$, respectively. Since the problem discussed is quasi-coupled, we will prove the following theorems and lemma: 
Theorem 1. Let $A^{\prime} \in U_{a d}^{\prime}, A_{n}^{\prime} \rightarrow A^{\prime}$ in $R^{p_{1}}$ as $n \rightarrow \infty$. Then

$$
T\left(A_{n}^{\prime}\right) \rightarrow T(A) \text { in } W_{1} .
$$

Sketch of the proof: Since

$$
b(A ; z, z) \geq\left(\min _{\iota \leq s, j \leq r^{\iota}} c_{\kappa}^{\iota}(j)\right) \sum_{\iota \leq s} \int_{\Omega^{\iota}} \mid \operatorname{grad} z^{\left.\iota\right|^{2}} d \mathbf{x},
$$

for $T_{n}:=T\left(A_{n}^{\prime}\right)$ we obtain $\left\|T_{n}\right\|_{W_{1}} \leq c$ for all $n$. Then a $T \in W_{1}$ and a subsequence $\left\{T_{m}\right\} \subset\left\{T_{n}\right\}$ exist such that

$$
T_{m} \rightarrow T \text { weakly in } W_{1}
$$

By definition

$$
b\left(A_{m}^{\prime} ; T_{m}, z\right)=s\left(A_{m}^{\prime} ; z\right) \quad \forall z \in V_{1}, \forall m
$$

Since

$$
\begin{array}{r}
\left|b\left(A_{m}^{\prime} ; T_{m}, z\right)-b\left(A^{\prime} ; T, z\right)\right| \rightarrow 0, \text { as } m \rightarrow \infty, \\
\left|s\left(A_{m}^{\prime} ; z\right)-b\left(A^{\prime} ; z\right)\right| \rightarrow 0, \text { as } m \rightarrow \infty
\end{array}
$$

we prove that

$$
\begin{array}{r}
b\left(A_{m}^{\prime} ; T_{m}, z\right) \rightarrow b\left(A^{\prime} ; T, z\right) \text { as } m \rightarrow \infty \\
s\left(A_{m}^{\prime} ; z\right) \rightarrow s\left(A^{\prime} ; z\right) \text { as } m \rightarrow \infty .
\end{array}
$$

Then we pass to the limit with $m \rightarrow \infty$ in (45). Using (46), (47) we prove that $T=T\left(A^{\prime}\right)$ is a weak solution of thermal part of the problem. Since it is unique, the whole sequence $\left\{T_{n}\right\}$ tends $T\left(A^{\prime}\right)$ weakly in $W_{1}$.

Remark 2. It can be proved that $T_{m} \rightarrow T$ converges also strongly in $W_{1}$.

Lemma 2. If $A_{n}^{\prime \prime} \in U_{a d}, A_{n}^{\prime \prime} \rightarrow A^{\prime \prime}$ in $R^{p_{2}}$, and $\mathbf{u}_{n} \rightarrow u$ weakly in $W$, then

$$
\begin{array}{cl}
a\left(A_{n}^{\prime \prime} ; \mathbf{u}_{n}, \mathbf{v}\right) \rightarrow a\left(A^{\prime \prime} ; \mathbf{u}, \mathbf{v}\right) & \forall \mathbf{v} \in W, \\
S\left(A_{n}^{\prime \prime} ; \mathbf{u}_{n}, T\right) \rightarrow S\left(A^{\prime \prime} ; \mathbf{u}, T\right) & \forall T \in W_{1} .
\end{array}
$$

Sketch of the proof: The proof follows from the fact that

$$
\begin{aligned}
\left|a\left(A_{n}^{\prime \prime} ; \mathbf{u}_{n}, \mathbf{v}\right)-a\left(A^{\prime \prime} ; \mathbf{u}, \mathbf{v}\right)\right| \rightarrow 0 \text { for } n \rightarrow \infty \\
\left|S\left(A_{n}^{\prime \prime} ; \mathbf{u}_{n}, T\right)-S\left(A^{\prime \prime} ; \mathbf{u}, T\right)\right| \rightarrow 0 \text { for } n \rightarrow \infty
\end{aligned}
$$


Theorem 2. Let $A_{n} \in U_{a d}, A_{n} \rightarrow A$ in $U \equiv R^{p_{2}}$. Then

$$
\mathbf{u}\left(A_{n}\right) \rightarrow \mathbf{u}(A) \quad \text { in } W
$$

Sketch of the proof: Let us denote $\mathbf{u}_{n}:=\mathbf{u}\left(A_{n}\right), \mathbf{u}:=\mathbf{u}(A), \mathbf{u}_{0 n}:=\mathbf{u}_{0}\left(A_{n}\right)$, $\mathbf{u}_{0}:=\mathbf{u}_{0}(A), T_{n}:=T\left(A_{n}\right), T:=T(A)$. Inserting $\mathbf{u}:=\mathbf{u}_{0}+\mathbf{w}(A), \mathbf{w}(A) \in K$, $\mathbf{u}_{n}:=\mathbf{u}_{0 n}+\mathbf{w}_{n}(A), \mathbf{w}_{n}(A) \in K, \mathbf{v}:=\mathbf{u}_{0}+\mathbf{w}$ or $\mathbf{v}:=\mathbf{u}_{0 n}+\mathbf{w}, \mathbf{w} \in K$ into the variational inequality $(10)$, we obtain

$$
a\left(A_{n} ; \mathbf{w}_{n}, \mathbf{w}-\mathbf{w}_{n}\right) \geq S\left(A_{n} ; \mathbf{w}-\mathbf{w}_{n}, T_{n}\right)-a\left(A_{n} ; \mathbf{u}_{0 n}, \mathbf{w}-\mathbf{w}_{n}\right) .
$$

Hence, putting $\mathbf{w}=0$, using Lemma 1 , Theorem 1 , definition of $U_{a d}^{u_{0 i}}$, after some modifications we find that

$$
c_{0}\left\|w_{n}\right\|_{W}^{2} \leq c_{7}\left\|w_{n}\right\|_{W}+c_{8} .
$$

As a consequence, $\mathbf{w}_{n}$ are bounded in $W$ and there exists a subsequence $\left\{\mathbf{w}_{k}\right\}$ and a function $\boldsymbol{\omega} \in W$ such that

$$
\mathbf{w}_{k} \rightarrow \boldsymbol{\omega} \quad \text { weakly in } W \text {, as } k \rightarrow \infty .
$$

It can be shown that $\omega=\mathbf{w}(A)$. Thus, since $\boldsymbol{\omega} \in K$ and since $a\left(A_{k} ; \mathbf{w}_{k}-\boldsymbol{\omega}, w_{k}-\right.$ $\boldsymbol{\omega}) \geq 0$, after some modification and using Lemma 2 , we obtain $\lim \inf a\left(A_{k} ; \mathbf{w}_{k}, \mathbf{w}_{k}-\right.$ $\boldsymbol{\omega}) \geq \lim a\left(A_{k} ; \boldsymbol{\omega}, \boldsymbol{w}_{k}-\boldsymbol{\omega}\right)=0$. Inserting $\mathbf{w}:=\boldsymbol{\omega}$ into (51) we arrive at

$$
a\left(A_{k} ; w_{k}, \omega-w_{k}\right) \geq S\left(A_{k} ; \omega-w_{k}, T_{k}\right)-a\left(A_{k} ; u_{0 k}, \omega-w_{k}\right)
$$

and

$\limsup a\left(A_{k} ; \mathbf{w}_{k}, \mathbf{w}_{k}-\boldsymbol{\omega}\right) \leq \limsup S\left(A_{k} ; \mathbf{w}_{k}-\boldsymbol{\omega}, T_{k}\right)+\lim \sup a\left(A_{k} ; \mathbf{u}_{0 k}, \boldsymbol{\omega}-\mathbf{w}_{k}\right)$.

For any $A \in U_{a d}, T \in W_{1}$ we can show that $\lim S\left(A_{k} ; \mathbf{w}_{k}-\boldsymbol{\omega}, T_{k}\right)=0$ and $\lim a\left(A_{k} ; \mathbf{w}_{k}, \mathbf{w}_{k}-\boldsymbol{\omega}\right)=0$ as $\lim \sup a\left(A_{k} ; \mathbf{w}_{k}, \mathbf{w}_{k}-\boldsymbol{\omega}\right) \leq 0$, from which it follows that $\lim a\left(A_{k} ; \mathbf{w}_{k}, \mathbf{w}_{k}-\boldsymbol{\omega}\right)=0$. It can be shown that $\mid a\left(A_{k} ; \mathbf{w}_{k}, \mathbf{w}-\right.$ $\left.\mathbf{w}_{k}\right)-a(A ; \boldsymbol{\omega}, \mathbf{w}-\boldsymbol{\omega}) \mid \rightarrow 0 ;$ then

$$
\lim a\left(A_{k} ; w_{k}, w-w_{k}\right)=a(A ; \omega, w-\omega)
$$

and since $\left|S\left(A_{k} ; \mathbf{w}-\mathbf{w}_{k}, T_{k}\right)-S(A ; \mathbf{w}-\boldsymbol{\omega}, T)\right| \rightarrow 0$, then

$$
\lim S\left(A_{k} ; w-w_{k}, T_{k}\right)=S(A ; w-\omega, T) .
$$

Moreover, we have $\left|a\left(A_{k} ; \mathbf{w}-\mathbf{w}_{k}, \mathbf{u}_{0 k}\right)-a\left(A ; \mathbf{w}-\boldsymbol{\omega}, \mathbf{u}_{0 k}\right)\right| \rightarrow 0$, where Lemma 1 , Lemma 2 and the convergence $\mathbf{u}_{0 k} \rightarrow \mathbf{u}_{0}$ in $W$ were used. Thus

$$
\lim a\left(A_{k} ; \mathbf{w}-\mathbf{w}_{k}, \mathbf{u}_{0 k}\right)=a\left(A ; \mathbf{w}-\boldsymbol{\omega}, \mathbf{u}_{0}\right) .
$$

Passing to the limit with $k \rightarrow \infty$, we obtain 


$$
a(A ; \boldsymbol{\omega}, \mathbf{w}-\boldsymbol{\omega}) \geq S(A ; \mathbf{w}-\boldsymbol{\omega}, T)-a\left(A ; \mathbf{w}-\boldsymbol{\omega}, u_{0}\right) .
$$

Since the variational inequality (10) has a unique solution, $\boldsymbol{\omega}=\mathbf{w}(A)$ follows from (53) and moreover, the whole sequence $\left\{\mathbf{w}\left(A_{n}\right)\right\}$ tends to $\mathbf{w}(A)$ weakly in $W$.

Furthermore, the strong convergence can also be proved.

\section{Existence of a Solution of the Worst Scenario Problem}

To prove the existence of a solution of the worst scenario problem, we will use the following lemma.

\section{Lemma 3.}

(i) Let $\Phi_{i}(T), i=1,2$, be defined by (35), (36) and let $T_{n} \rightarrow T$ in $W_{1}$, as $n \rightarrow \infty$. Then

$$
\lim _{n \rightarrow \infty} \Phi_{i}\left(T_{n}\right)=\Phi_{i}(T), \quad i=1,2 .
$$

(ii) Let $\Phi_{i}(\mathbf{u}), i=3,4$, be defined by (37), (38) and let $\mathbf{u}_{n} \rightarrow \mathbf{u}$ in $W$, as $n \rightarrow \infty$. Then

$$
\lim _{n \rightarrow \infty} \Phi_{i}\left(\mathbf{u}_{n}\right)=\Phi_{i}(\mathbf{u}), \quad i=3,4
$$

(iii) Let $\Phi_{i}(A ; \mathbf{u}, \mathbf{T}), i=5,6$, be defined by (39), (40) and let $A_{n} \rightarrow A$ in $U$, $A_{n} \in U_{a d}, \mathbf{u}_{n} \rightarrow \mathbf{u}$ in $W$ and $T_{n} \rightarrow T$ in $L^{2}(\Omega)$, as $n \rightarrow \infty$. Then

$$
\lim _{n \rightarrow \infty} \Phi_{i}\left(A_{n}, \mathbf{u}_{n}, T_{n}\right)=\Phi_{i}(A, \mathbf{u}, T), \quad i=5,6
$$

The proof is a modification of that of [3].

As the main result of the paper we present the following theorem:

Theorem 3. There exists at least one solution of the worst scenario problems (41), (42), $i=1, \ldots 6$.

The proof is a modification of that of [3].

\section{Conclusion}

Mathematical models connected with the safety of construction and of operation of the radioactive waste repositories involve input data (thermal conductivity and elastic coefficients, body and surface forces, thermal sources, coefficients of thermal expansion, boundary values, coefficient of friction on contact boundaries, etc.) which cannot be determined uniquely, but only in some intervals, given by the accuracy of measurements and the approximate solutions 
of identification problems. The notation "reliable solution" denotes the worst case among a set of possible solutions where the degree of badness is measured by a criterion functional. For the safety of the radioactive waste repositories we seek the maximal value of this functional, which depends on the solution of the mathematical model. Then for the computations of such problems (some mean values of temperatures, displacements, intensity of shear stresses, principal stresses, stress tensor components, normal and tangential components of the displacement or stress vector on the contact boundaries, etc.) we have to formulate a corresponding maximization (worst scenario) problem. Then methods and algorithms known from "optimal design" can be used.

To construct a model of structures under the influence of critical conditions the influence of global tectonics onto a local area, where the critical structure is built as well as the influence of the resulting local geomechanical processes on a critical structure must be taken into account ([6]). Problems of this kind with uncertain input data are problems with high level radioactive waste repositories. In the case of the high level radioactive waste repositories the effects of geodynamical processes in the sense of plate tectonics must be taken into consideration, namely in regions near tectonic areas (e.g. the Japan island arc, the Central and South Europe, etc), but also in the platform regions (as in Sweden, Canada, etc.). Another example is represented by modelling an interaction between a tunnel wall and a rock massif in the radioactive waste repository tunnels or by modelling of a tunnel crossing by an active deep fault(s), respectively.

\section{Acknowledgements}

The first author thankfully acknowledges the support of the Grant Agency of the Czech Republic under the grant 201/01/1200. Both authors acknowledge the support under the grant COPERNICUS-HIPERGEOS II INCO-KIT-977006 and the grant of the Ministry of Education, Youth and Sports of the Czech Republic No OK-407.

The paper is prepared for the workshop on "Numerical Models in Geomechanics" of the conference ICCS'2002, Amsterdam, April 2002 and will be published in the Springer Lecture Notes in Computer Science.

\section{References}

1. Hlaváček, I.: Reliable Solution of a Signorini Contact Problem with Friction, Considering Uncertain Data. Numer. Linear Algebra Appl. 6 (1999) 411-434

2. Hlaváček, I., Nedoma, J.: On a Solution of a Generalized Semi-Coercive Contact Problem in Thermo-Elasticity. Mathematics and Computers in Simulation (in print) (2001)

3. Hlaváček, I., Nedoma, J.: Reliable Solution of a Unilateral Contact Problem with Friction and Uncertain Data in Thermo-Elasticity (to apperar) (2002)

4. Nečas, J., Hlaváček, I.: Mathematical Theory of Elastic and Elasto-Plastic Bodies: An Introduction. Elsevier, Amsterdam (1981) 
5. Nedoma, J.: On the Signorini Problem with Friction in Linear Thermo-Elasticity. The quasi-coupled 2D-case. Appl.Math. 32 (3) (1987) 186-199

6. Nedoma, J.: Numerical Modelling in Applied Geodynamics. John Wiley\&Sons, Chichester, New York, Weinheim, Brisbane, Singapore, Toronto (1998)

7. Nedoma, J., Haslinger, J., Hlaváček, I.: The Problem of an Obducting Lithospheric Plate in the Aleutian Arc System. A Finite Element Analysis in the Frictionless Case. Math. Comput. Model. 12 (1989) 61-75

8. Rohn, J.: Positive Definiteness and Stability of Interval Matrices. SIAM J. Matrix Anal. Appl. 15 (1994) 175-184 